\title{
Monsoon and general circulation system
}

\author{
BERGER André \\ Institut d'Astronomie et de Géophysique G. Lemaître, Université Catholique de Louvain, Chemin du Cyclotron 2, 1348 Louvain-la- \\ Neuve, Belgium
}

The instrumental period of meteorological record is too short to capture the full variability of the climate system and, in particular to visualize the type of climate that is predicted to occur over the next decades and centuries. It is therefore important to reconstruct past climates and understand past climatic variations. Among them, those related to the monsoon appear to be more and more important for Society. The economy, culture and rhythms of life of a large part of humanity are critically influenced by the evolution and variability of the monsoon. In particular, the Asian monsoon is one of the most spectacular occurrences in the climate system, strongly influenced by the combination of the thermal contrast between the Eurasian continent and the Indo-Pacific Ocean and the presence the Tibetan Plateau. Climatologically, the monsoon regions as the most convectively active areas, are situated in the Intertropical Convergence Zone and account for the majority of global atmospheric heat and moisture transport ${ }^{[1]}$. The need to better understand the monsoon leads inevitably to the close inspection of its worldwide activity during the geological times to provide a long-term perspective from which any future change may be more effectively assessed.

It is in that framework that the substantial paper by Wang Pinxian in this issue ${ }^{[2]}$ is more than welcome. It is an authoritative review, and probably also the first in which the monsoon issues are reviewed in a global scale through a so long geological history. Wang's main points are fundamental, and hence important. First, it is to emphasize the role of the tropics as an extremely important source of changes in the global climate system. Second, it is to regard the monsoons as a global feature through which the tropical forcing exerts its climatic role worldwide. I totally agree with these arguments, and share
Wang's concern that the climate and paleoclimate community has, since too long, viewed the monsoons as local phenomena, without trying enough to relate their behavior together. But the problem being extremely complex, let me take the opportunity to put it in its broad context.

The reader will find an interesting brief introduction to monsoon, its origin, history and localization in http://en.wikipedia.org/wiki/Monsoon. Etymologically, monsoon comes from the Arabic word mawsim, which means season, explaining why the original definition includes only major wind systems that change direction seasonally. The most spectacular occurrence of such seasonally varying circulation is undoubtedly in Southern and Eastern Asia. It is the associated heavy rainfall there, which has allowed by extension the other regions of the world to qualify as monsoon regions. With time, the term monsoon has been broadened to include almost all of the phenomena associated with the seasonal weather cycle within tropical and subtropical land regions of the Earth, showing immediately the relevance of Wang's argumentation to deal with monsoon as a global tropical system.

Giving stronger emphasis to the tropical forcing does not mean that we can isolate the tropical climate from the rest of the world, as it is part of the Earth's climate system. The response of the climate system to the energy that we receive from the Sun (both at the seasonal and geological time scales) involves all latitudes and seasons and depends upon the feedback mechanisms inherent in the climate system itself. Among them, the water vapor

Received February 11, 2009; accepted February 12, 2009

doi: 10.1007/s11434-009-0170-y

email: andre.berger@uclouvain.be 
feedback is particularly efficient in the intertropical zone which, in addition, receives half of the energy sent by the Sun. It therefore can certainly not be ignored in the discussion. The other feedback is the albedo-temperature one, which is significant in the high latitudes, covered by snow and ice. The amplitude of its seasonal cycle is so large - mainly in the Northern Hemisphere - that it can not either be ignored, especially in problems where seasonality is important, like in the atmospheric general circulation. An example of the complex, rather unexpected, is the possible relationship between high latitude ice sheets and precipitation over East China ${ }^{[3]}$. The surface albedo in the polar latitudes influences indeed the magnitude and seasonal behavior of the latitudinal gradient in net radiation which, with the rotation of the Earth, drives the three-cell regime observed in the mean meridional circulation ${ }^{[1]}$. The Hadley cells tend therefore to fluctuate in intensity and locations with the seasons, being strongest in the winter hemisphere, and so does the ITCZ, a band of organized convection reflecting the convergence of surface air from the two hemispheres inside the Hadley circulations. This ITCZ, which migrates north and south annually with the Sun and involves the monsoon particularly over southeastern Asia and northern Australia during the solstices, is therefore strongly linked to the high latitudes and not only to the tropics. Finally, this movement of the ITCZ is also associated to the largest seasonal pressure variations which are found over the Asian continent where a strong anticyclone develops over Siberia during winter and a low-pressure system forms during summer north of the Indian subcontinent. This implies that the longitudinal gradient of pressure (and temperature) between the Asian continent and the Pacific and Indian oceans cannot be ignored either.

As far as the astronomical theory is concerned, I share Wang's view ${ }^{[2]}$ that the astronomical signal in paleoclimate data is very helpful for better understanding the

1 Peixioto J P, Oort A H, Physics of Climate. New York: American Institute of Physics, 1993. 520

2 Wang P X. Global monsoon in a geological perspective. Chin Sci Bull, 2009, 54(7): 1113-1136

3 Yin Q, Berger A, Driesschaert E, et al. The Eurasian ice sheet reinforces the East Asian summer monsoon during the interglacial 500000 years ago. Clim Past, 2008, 4: 79-90

4 Berger A. Long-term variations of daily insolation and Quaternary climatic changes. J Atmos Sci, 1978, 35(12): 2362-2367

5 Berger A, Mélice J L, Loutre M F. On the origin of the 100-kyr cy- physical mechanisms driving the historical behavior of the monsoon. Looking for the astronomical frequencies in proxy records is certainly the first knowledgeable step. Their list is available in Berger $(1978)^{[4]}$ who was the first to calculate them, in Berger et al. (2005) ${ }^{[5]}$ where more details can be found about the origin of the $100 \mathrm{ka}$ cycle and in Berger et al. ${ }^{[6]}$ where precession harmonics are shown to exist in equatorial insolation. The next step is then to analyze the relationship between the insolation forcing and the climate system response. Here, we must also be very careful in choosing the insolation values. As shown in Berger and Pestiaux (1984) ${ }^{[7]}$, the total energy received during a season is only a function of obliquity whereas the length of the seasons is only a function of precession. Combination of both signals characterizes the behavior of the average seasonal insolation and of the daily insolation in general. This is important to consider because the time behavior of these different insolation parameters differs also significantly from one to the others $^{[8]}$

Beside the global scope, one of the highlights of Wang's review ${ }^{[2]}$ is the attempt to trace back to the deeper geological past. With the pre-Quaternary records of climate, it is effectively possible to address the long-term orbital cycles, and to examine the monsoon behaviors at the time when the North or both poles were ice-free. Although the data limitation currently precludes firm conclusions, it is worthy noting Wang's idea on if the long eccentricity signals in the climate system were related to long-term monsoon cycles.

There would be a long way to go for the science community to understand the interactions between the tropical forcing and high-latitude forcing. Towards this end, I totally agree with Wang's argumentation about paying more attention to the importance of the tropical forcing in modulating the Earth's climate system. It is rightly this kind of efforts that are beguiling mixture of coherence and debate to keep us all on our toes.

cles in the astronomical forcing. Paleoceanography, 2005, 20, PA4019, DOI: 10.1029/2005PA001173

6 Berger A, Loutre M F, Mélice J L, et al. Equatorial insolation: from precession harmonics to eccentricity frequencies. Clim Past, 2, 2006. $131-136$

7 Berger A, Pestiaux P. Accuracy and stability of the Quaternary insolation. In: Milankovitch and Climate. Berger A, Imbrie J, Hays J, et al. NATO ASI Series C, Vol. 126, Dordrecht: D. Reidel Publishing Company, 1984

8 Berger A, Loutre M F, Tricot Ch. Insolation and Earth's orbital periods. J Geophys Res, 1993, 98(D6): 10341-10362 